\title{
N-cadherin, a novel prognostic biomarker, drives malignant progression of colorectal cancer
}

\author{
XUEBING YAN ${ }^{*}$, LEILEI YAN*, SIHONG LIU, ZEZHI SHAN, YUAN TIAN and ZHIMING JIN \\ Department of Surgery, Sixth People's Hospital, School of Medicine, \\ Shanghai Jiao Tong University, Shanghai 200233, P.R. China
}

Received May 15, 2014; Accepted February 6, 2015

DOI: $10.3892 / \mathrm{mmr} .2015 .3687$

\begin{abstract}
Recent studies have indicated that the epithelial-mesenchymal transition (EMT) is a key molecular mechanism involved in the development of colorectal cancer (CRC). N-cadherin is a mesenchymal marker of the EMT and has been closely linked to several human malignancies. However, its role in CRC has remained elusive. In the present study, qRT-PCR and western blot analysis indicated that $\mathrm{N}$-cadherin expression was higher in tumor tissues than in that in their adjacent normal tissues. Immunohistochemical evaluation of $\mathrm{N}$-cadherin and $\mathrm{E}$-cadherin (an epithelial marker of the EMT), indicated that N-cadherin expression was significantly associated with tumor differentiation, tumor size as well as tumor, nodes and metastasis stage. Correlation analysis suggested the expression of $\mathrm{N}$-cadherin was negatively correlated with that of E-cadherin in CRC tissues. Kaplan-Meier analysis indicated that patients with high $\mathrm{N}$-cadherin expression had a significantly lower overall survival and disease-free survival rate than those with low $\mathrm{N}$-cadherin expression, while the opposite was found for E-cadherin. Of note, the present study found that high $\mathrm{N}$-cadherin expression was an independent prognostic factor for CRC. In vitro assays showed that $\mathrm{N}$-cadherin was widely expressed in CRC cell lines and silencing of $\mathrm{N}$-cadherin suppressed the proliferation and migration of the CRC cell line HT-29 by upregulating E-cadherin, suggesting a potential role of $\mathrm{N}$-cadherin in inducing EMT. In conclusion, the present study suggested that $\mathrm{N}$-cadherin has the potential of serving as a novel prognostic predictor and a promising therapeutic target for CRC.
\end{abstract}

Correspondence to: Dr Zhiming Jin, Department of Surgery, Sixth People's Hospital, School of Medicine, Shanghai Jiao Tong University, 600 Yishan Road, Shanghai 200233, P.R. China

E-mail: jzmgyp@aliyun.com

*Contributed equally

Key words: N-cadherin, prognosis, epithelial-mesenchymal transition, colorectal cancer

\section{Introduction}

Colorectal cancer (CRC) is the third most common cancer in males and the second most common cancer in females, accounting for 1.2 million novel cancer cases and 608,700 mortalities worldwide in 2008 (1). According to the American Cancer Society, 136,830 novel cases and 50,310 mortalities from CRC were estimated for 2014 in the United States (2). Despite improved medical treatments and increased awareness for early detection, approximately $25 \%$ of CRC patients present with distant metastases at initial diagnosis and nearly $50 \%$ will develop metastases (3). Moreover, the relative five-year survival rate of CRC patients with metastases is only $10 \%$ (4). It has been widely accepted that conventional therapeutic strategies are helpful for clinical treatment; however, they are far from sufficient. Deficiencies of the current therapies for CRC may be partly due to the limited understanding of its underlying molecular mechanisms.

It is now well established that a developmental regulatory program, known as the epithelial-mesenchymal transition, (EMT), broadly participates in invasion and metastasis of neoplastic malignancies (5). Through the EMT program, cancer cells can acquire enhanced abilities to resist apoptosis, to invade and to migrate. The EMT program is also known as a cadherin switching process leading to loss of E-cadherin and gain of $\mathrm{N}$-cadherin (6). E-cadherin, a transmembrane protein mediating cell-cell adhesion, has been widely known as a tumor suppressor and the best-characterized molecular marker of the EMT (7). Loss of E-cadherin has been reported to exert an unfavorable impact on the overall survival rate of CRC patients (8). However, N-cadherin, a transmembrane protein similar to E-cadherin, has been reported to be abnormally expressed in multiple tumors, including pancreatic (9), gastric (10) and hepatocellular (11) cancers. High expression of $\mathrm{N}$-cadherin predicts poor outcome in patients with superficial urothelial carcinoma and gallbladder cancer $(12,13)$. Moreover, cellular studies have suggested that $\mathrm{N}$-cadherin is able to drive numerous hallmarks of cancer, including proliferation (14), invasion (15), metastasis (16) and resistance to chemotherapy (17).

Despite the increasing number of studies on N-cadherin, its role in CRC has remained elusive. The present study demonstrated for the first time, to the best of our knowledge, that high expression of $\mathrm{N}$-cadherin may be associated with the 
malignant progression of $\mathrm{CRC}$ and predict poor prognosis in CRC patients. Furthermore, in vitro RNA interference techniques were used to investigate the role of $\mathrm{N}$-cadherin in the proliferation and migration, and therefore, the EMT of CRC cells.

\section{Materials and methods}

Tissue samples and patient data. In the present study, a total of 23 fresh primary CRC tissues and their adjacent normal tissues were prepared for quantitative real-time polymerase chain reaction and western blot analyses. In addition, a total of 102 paraffin-embedded primary CRC tissues and their adjacent normal tissues were prepared for immunohistochemical analysis. The specimens were collected from patients with CRC undergoing surgery at Department of Surgery, The Sixth People's Hospital affiliated to Shanghai Jiao Tong University (Shanghai, China). Table I shows the basic clinical characteristics of patients in the present study. None of the patients had received pre-operative chemotherapy or radiotherapy. Overall survival (OS) is defined as the interval from the date of surgery to the date of death, or the date of the last follow-up. Disease-free survival (DFS) is defined as the interval from the date of surgery to the date of tumor recurrence and progression. Prior to using tissue specimens in the present study, approval by the ethics committee of the Sixth People's Hospital affiliated to Shanghai Jiao Tong University and written informed consent from patients were obtained.

Reverse transcription quantitative polymerase chain reaction (RT-qPCR). Total RNA was isolated using TRIzol (Invitrogen Life Technologies, Carlsbad, CA, USA) according to the manufacturer's instructions. The isolated RNA was reversely transcribed into cDNA by Superscript III Reverse Transcriptase (Promega, Madison, WI, USA). PCR amplification of the cDNA template was then performed using SYBR Green mix (TaKaRa, Otsu, Japan) using a StepOne Plus Real-Time PCR System (Applied Biosystems, Thermo Fisher Scientific, Waltham, MA, USA). The reaction conditions were as follows: $95^{\circ} \mathrm{C}$ for $30 \mathrm{sec}, 40$ cycles of $95^{\circ} \mathrm{C}$ for $5 \mathrm{sec}$ and $60^{\circ} \mathrm{C}$ for $30 \mathrm{sec}$. The reactions were repeated in triplicate and the data were normalized to $\beta$-actin. The products of RT-qPCR were electrophoresed on $2 \%$ agarose gel (BD Biosciences, San Jose, CA, USA) using Tris/Boric Acid/EDTA buffer (BD Biosciences). An AlphaImager Gel Documentation system (ProteinSimple, San Jose, CA, USA) was then used to detect product size. The $2^{-\Delta \Delta \mathrm{T}}$ method was employed to calculate the relative expression levels of the genes. The sequences of PCR primers (Invitrogen Life Technologies) used in the present study were as follows: N-cadherin (CDH2) forward, 5'-CAAGATGGGTCAATGGAAATAG-3' and reverse, 5'-CTCAGGAATACGAGCCTTCAC-3'; E-cadherin (CDH1) forward, 5'-AAGACAAAGAAGGCA AGGT-3' and reverse, 5'-AGAGAGTGTATGTGGCAATG-3'; $\beta$-actin forward, 5'-AAGGTGACAGCAGTCGGTT-3' and reverse, 5'-TGTGTGGACTTGGGAGAGG-3'.

Western blot analysis. Protein extracted from fresh tissues was separated by $10 \%$ SDS-PAGE [JRDUN Biotechnology (Shanghai) Co., Ltd., Shanghai, China] and transferred to a nitrocellulose membrane (Merck Millipore, Billerica, MA, USA). The membrane was blocked with $5 \mathrm{~g}$ skimmed milk powder (BD Biosciences) in $100 \mathrm{ml}$ Tris-buffered saline [JRDUN Biotechnology (Shanghai) Co., Ltd.] containing 0.05\% Tween-20 (Amresco LLC, Solon, OH, USA) (TBST) for $1 \mathrm{~h}$ at room temperature. The membrane was then incubated with rabbit polyclonal anti-N-cadherin (1:5,000; cat. no. ab18203; Abcam, Cambridge, MA, USA) and rabbit polyclonal anti- $\beta$-actin (1:3,000; cat. no. P30002; Abmart, Shanghai, China) overnight at $4^{\circ} \mathrm{C}$. $\beta$-actin was used as an internal control. Following incubation with goat anti-rabbit horseradish peroxidase-conjugated immunoglobulin $(\mathrm{Ig}) \mathrm{G}$ (1:2,000; cat. no. M21002; Abmart) for $2 \mathrm{~h}$ at $37^{\circ} \mathrm{C}$, an enhanced chemiluminescence reagent (Santa Cruz Biotechnology, Inc., Dallas, TX, USA) was employed for protein detection. After incubation with enhanced chemiluminescence reagent for $5 \mathrm{~min}$, the membrane was visualized for $30 \mathrm{sec}$ using photographic film (Kodak, Rochester, NY, USA) in a cassette (Kodak). Finally, the film was incubated with developing solution (Shanghai Sanlian Group Co., Ltd., Shanghai, China) for 2 min and fixing solution (Shanghai Sanlian Group Co., Ltd.) for $1 \mathrm{~min}$.

Immunohistochemistry and staining evaluation. Paraffin-embedded tissue specimens were cut into 4- $\mu \mathrm{m}$ sections. The sections were deparaffinized in xylene and dehydrated in ethanol (Sinopharm Chemical Reagent Co., Ltd., Shanghai, China). Following antigen retrieval by microwave for $25 \mathrm{~min}, 0.3 \%$ hydrogen peroxide and methanol (Sinopharm Chemical Reagent Co., Ltd.) were used to block endogenous peroxidase activity. The sections were then incubated with rabbit polyclonal anti-N-cadherin (1:150) and rabbit monoclonal anti-E-cadherin (1:150; cat. no. ab40772; Abcam) overnight at $4^{\circ} \mathrm{C}$. After three washes with phosphate-buffered saline (PBS; Sinopharm Chemical Reagent Co., Ltd.), the sections were incubated with polyclonal goat anti-rabbit $\operatorname{IgG}\left(1: 250\right.$; cat. no. ab150077; Abcam) for $25 \mathrm{~min}$ at $37^{\circ} \mathrm{C}$. Finally, the sections were incubated with diaminobenzidine (Invitrogen Life Technologies) for $5 \mathrm{~min}$ and counterstained with hematoxylin (Invitrogen Life Technologies) for $2 \mathrm{~min}$. Sections incubated with PBS instead of primary antibody were utilized as negative controls.

Staining evaluation was performed by two independent researchers blinded to the clinicopathological characteristics of the patients. Five bright fields were randomly selected for evaluation. The semi-quantitative staining evaluation was strictly based on a product of Staining Intensity (SI) and Percentage of Positive cells (PP). SI was determined as follows: Negative (score 0), weak (score 1), moderate (score 2) and strong (score 3). PP was determined as follows: 0 (score 0), $\leq 10 \%$ (score 1), $11-50 \%$ (score 2), $51-80 \%$ (score 3 ), $>80 \%$ (score 4). A final staining score of 0-3 or 3-12 indicated low or high expression, respectively.

Cell culture. All the CRC cell lines (HCT-116, RKO, DLD-1, HT-29 and Caco-2) were purchased from the Type Culture Collection of the Chinese Academy of Sciences (Shanghai, China). The HCT-116 and HT-29 cell lines were maintained in McCoy'5a culture medium (Sigma-Aldrich, St Louis, MO, USA). The RKO and Caco-2 cell lines were maintained in MEM 
Table I. Correlation between N-cadherin expression and clinicopathological characteristics in patients with colorectal cancer.

\begin{tabular}{|c|c|c|c|c|}
\hline \multirow[b]{2}{*}{ Characteristic } & \multirow[b]{2}{*}{ Total (n) } & \multicolumn{2}{|c|}{$\mathrm{N}$-cadherin } & \multirow[b]{2}{*}{ P-value } \\
\hline & & High expression (n) & Low expression (n) & \\
\hline Gender & & & & 0.829 \\
\hline Male & 60 & 42 & 18 & \\
\hline Female & 42 & 28 & 14 & \\
\hline Age (years) & & & & 0.389 \\
\hline$\leq 60$ & 41 & 26 & 15 & \\
\hline$>60$ & 61 & 44 & 17 & \\
\hline Tumor location & & & & 0.392 \\
\hline Colon & 56 & 36 & 20 & \\
\hline Rectum & 46 & 34 & 12 & \\
\hline Tumor differentiation & & & & 0.007 \\
\hline Well & 35 & 18 & 17 & \\
\hline Moderate & 31 & 21 & 10 & \\
\hline Poor & 36 & 31 & 5 & \\
\hline Tumor size (cm) & & & & 0.031 \\
\hline$\leq 5$ & 60 & 36 & 24 & \\
\hline$>5$ & 42 & 34 & 8 & \\
\hline Tumor invasion & & & & 0.021 \\
\hline $\mathrm{T} 1$ & 11 & 5 & 6 & \\
\hline $\mathrm{T} 2$ & 34 & 19 & 15 & \\
\hline $\mathrm{T} 3$ & 42 & 35 & 7 & \\
\hline $\mathrm{T} 4$ & 15 & 11 & 4 & \\
\hline Lymph node metastasis & & & & 0.001 \\
\hline Present & 57 & 47 & 10 & \\
\hline Absent & 45 & 23 & 22 & \\
\hline Distant metastasis & & & & 0.010 \\
\hline Present & 49 & 40 & 9 & \\
\hline Absent & 53 & 30 & 23 & \\
\hline
\end{tabular}

culture medium (Sigma-Aldrich). The DLD-1 cell line was maintained in RPMI-1640 culture medium (Sigma-Aldrich). All culture media were supplemented with $10 \%$ fetal bovine serum (Gibco-BRL, Invitrogen Life Technologies) and 1\% penicillin/streptomycin (Gibco-BRL, Invitrogen Life Technologies) at $37^{\circ} \mathrm{C}$ in a $5 \% \mathrm{CO}_{2}$ humidified atmosphere.

Small interfering RNA transfection. Small interfering RNA (siRNA) for N-cadherin (Invitrogen Life Technologies) was employed to down-regulate the expression of $\mathrm{N}$-cadherin in cell lines as described previously $(14,17)$. The siRNA sequence was 5'-CUAACAGGGAGUCAUAUGGUGGAGC-TdT-3'. A negative control siRNA was also purchased from Invitrogen Life Technologies and was used for minimizing nonspecific effects. The sequence of the control siRNA was 5'-TTCTCCGAACGTGTCACGT-3'. siRNA transfection was performed with Lipofectamine 2000 transfection reagent (Invitrogen Life Technologies) according to the manufacturer's instructions. 24-48 h after transfection, the cells were collected for the subsequent assays.
MTT assay. The MTT assay was employed to determine the growth of cells following transfection. Briefly, the cells were seeded into 96 -well plates at a density of $3 \times 10^{3}$ cells per well. 24, 48 and $72 \mathrm{~h}$ following transfection, $150 \mu \mathrm{l}$ culture medium containing $0.5 \mathrm{mg} / \mathrm{ml} \mathrm{MTT}$ was added into each well and the cells were incubated at $37^{\circ} \mathrm{C}$ for $4 \mathrm{~h}$. Following removal of the culture medium, DMSO was added to dissolve the purple precipitate. Finally, the absorbance at a wavelength of $490 \mathrm{~nm}$ was measured using a SpectraMax 340PC384 microplate reader (Molecular Devices, Sunnyvale, CA, USA). The assay was repeated three times independently.

Wound healing assay. To assess cell migration, the wound healing assay was performed according to a method by Liang et al (18). Briefly, the cells were cultured in each well to reach $90 \%$ confluence. Following aspiration of the medium, a sterile pipette tip was used to create a wound in the cell monolayer. The cells were then washed twice and re-cultured with serum-free medium. Following $24 \mathrm{~h}$, the wound was monitored and photographed by a CX41 biological microscope 
(Olympus Corporation, Tokyo, Japan). The assay was repeated three times independently.

Statistical analysis. All values are presented as the mean \pm standard deviation. SPSS 17.0 statistical software (SPSS, Inc., Chicago, IL, USA) was used for statistical analysis. Differences between two groups were determined using the two-tailed Student's t-test. Correlations between N-cadherin expression and clinicopathological parameters were determined using the $\chi^{2}$ test, while the correlation between $\mathrm{N}$-cadherin and E-cadherin expression was determined by a non-parametric Spearman's rank correlation coefficient. Survival curves were generated using the Kaplan-Meier model and the difference between the survival of subgroups was compared using the log-rank test. Significant independent prognostic factors were identified by multivariate analysis based on the Cox proportional hazard model. A P-value $<0.05$ was considered to indicate a statistically significant difference between values.

\section{Results}

$N$-cadherin expression in CRC tissues and adjacent normal tissues. Firstly, the mRNA expression of $\mathrm{N}$-cadherin was assessed in CRC tissues and adjacent normal tissues. The results showed that the $\mathrm{N}$-cadherin mRNA expression was upregulated in 19 of the $23 \mathrm{CRC}$ tissues compared with those in adjacent normal tissues. As shown in Fig. 1A, the relative mean expression of $\mathrm{N}$-cadherin mRNA in CRC tissues $(1.24 \pm 0.42)$ was significantly higher than that in adjacent normal tissues $(0.44 \pm 0.27, \mathrm{P}=0.0028)$. Western blot analysis showed that $\mathrm{N}$-cadherin protein levels were similar to mRNA levels in the CRC and adjacent normal tissues (Fig. 1B). Moreover, immunohistochemical analysis was performed to detect protein expression of $\mathrm{N}$-cadherin in 102 paraffin specimens. The results showed that high expression of $\mathrm{N}$-cadherin was present in $68.6 \%$ (70/102) of CRC tissues. Figure 2 shows representative results of immunohistochemical staining for $\mathrm{N}$-cadherin.

Correlation between the protein expression of $\mathrm{N}$-cadherin and clinicopathological parameters. Correlations between the protein expression of $\mathrm{N}$-cadherin and clinicopathological parameters are summarized in Table I. N-cadherin expression was significantly correlated with tumor differentiation ( $\mathrm{P}=0.007)$, tumor size $(\mathrm{P}=0.031)$, tumor invasion $(\mathrm{P}=0.021)$, lymph node metastasis $(\mathrm{P}=0.001)$ and distant metastasis $(\mathrm{P}=0.010)$. However, $\mathrm{N}$-cadherin expression was not correlated with other parameters, including gender $(\mathrm{P}=0.829)$, age $(\mathrm{P}=0.389)$ and tumor location $(\mathrm{P}=0.392)$.

Expression of E-cadherin and its correlation with $N$-cadherin in CRC tissues. Since previous studies showed that N-cadherin is involved in the EMT in certain tumor types and E-cadherin is the best-characterized molecular marker of the EMT, the present study performed immunohistochemical staining for E-cadherin in CRC tissues and investigated its possible association with N-cadherin (Fig. 2; Table II). According to the established evaluation principles applied, high expression of E-cadherin was only detected in 37.3\% (38/102) of CRC tissues. Moreover, correlation analysis suggested that the
$\mathbf{A}$
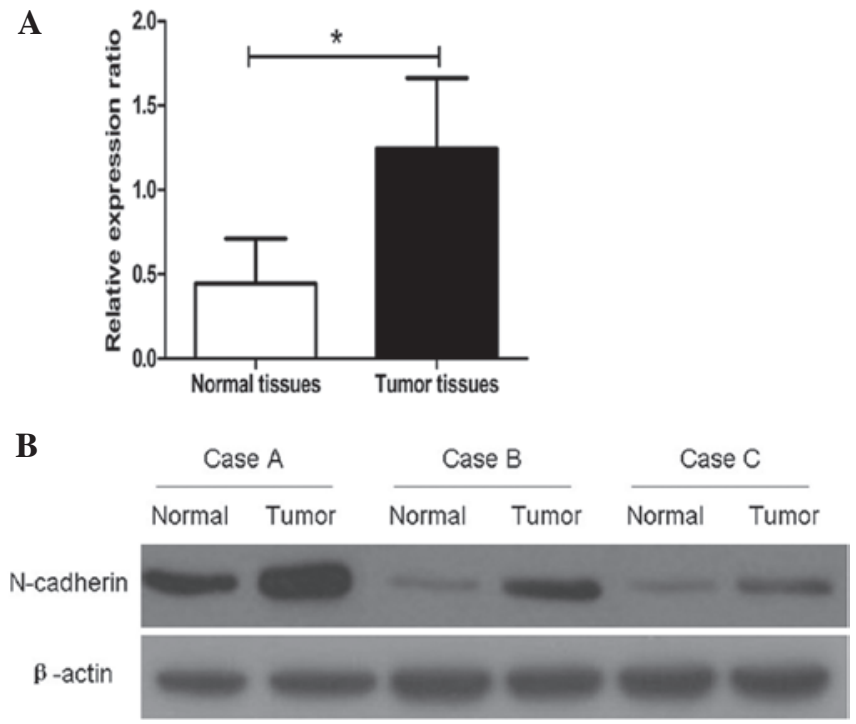

Figure 1. Expression of $\mathrm{N}$-cadherin in $\mathrm{CRC}$ tissues and their adjacent normal tissues. (A) mRNA expression of $\mathrm{N}$-cadherin in CRC tissues was significantly higher than that in their adjacent normal tissues $(1.24 \pm 0.42$ vs. $0.44 \pm 0.27$, ${ }^{*} \mathrm{P}=0.0028$ ). (B) Western blot analysis also showed that the protein expression of N-cadherin was up-regulated in CRC tissues compared with that in their adjacent normal tissues. CRC, colorectal cancer.

expression of $\mathrm{N}$-cadherin and $\mathrm{E}$-cadherin were negatively correlated ( $r=-0.528$, P $<0.001$; Table II). Fig. 2 shows representative results of immunohistochemical staining for E-cadherin.

Prognostic significance of $\mathrm{N}$-cadherin and $\mathrm{E}$-cadherin in $\mathrm{CRC}$ patients. The association between the protein expression and prognosis for CRC patients was investigated by Kaplan-Meier survival analysis (Fig. 3). The log-rank test indicated that the OS rate of patients with high $\mathrm{N}$-cadherin expression was significantly lower than that in patients with low N-cadherin expression (Fig. 3A, $\mathrm{P}=0.021$ ). However, the OS rate of patients with high E-cadherin expression was significantly higher than that of patients with low E-cadherin expression (Fig. 3B, $\mathrm{P}=0.018)$. Similar results were also observed in the DFS survival analysis (N-cadherin, $\mathrm{P}=0.020$, Fig. 3C; E-cadherin, $\mathrm{P}=0.014$, Fig. 3D).

As shown in Table III, univariate analysis suggested that $\mathrm{N}$-cadherin expression, E-cadherin expression, tumor differentiation, tumor invasion, lymph node metastasis and distant metastasis were significant prognostic factors for $\mathrm{CRC}$ patients ( $\mathrm{P}=0.001, \mathrm{P}=0.014, \mathrm{P}=0.041, \mathrm{P}=0.033, \mathrm{P}=0.030$ and $\mathrm{P}=0.006$, respectively), while other clinicopathological parameters such as gender and age were not. Moreover, multivariate analysis indicated that $\mathrm{N}$-cadherin expression, E-cadherin expression and distant metastasis were independent prognostic factors for CRC patients $(\mathrm{P}=0.012, \mathrm{P}=0.028$ and $\mathrm{P}=0.026$, respectively).

Silencing $\mathrm{N}$-cadherin inhibits proliferation and migration of $C R C$ cells by reversing the EMT. The mRNA expression of $\mathrm{N}$-cadherin in CRC cell lines was detected by RT-qPCR. As shown in Fig. 4A, N-cadherin was expressed in all CRC cell lines, while expression was significantly higher in the HT-29 cell line than that in the other cell lines assessed (HT-29 vs. HCT116, $\mathrm{P}=0.0042$; HT-29 vs. RKO, $\mathrm{P}=0.0147$; HT-29 vs. DLD-1, $\mathrm{P}=0.0049$; HT-29 vs. Caco-2, $\mathrm{P}=0.0004$ ). 
Table II. Correlation between N-cadherin expression and E-cadherin expression in colorectal cancers.

\begin{tabular}{|c|c|c|c|c|c|}
\hline \multirow[b]{2}{*}{$\mathrm{N}$-cadherin expression } & \multirow[b]{2}{*}{ Total (n) } & \multicolumn{2}{|c|}{ E-cadherin } & \multirow[b]{2}{*}{$\mathrm{r}$} & \multirow[b]{2}{*}{ P-value } \\
\hline & & Low expression $(\mathrm{n})$ & High expression (n) & & \\
\hline Low & 32 & 8 & 24 & -0.528 & $<0.001$ \\
\hline High & 70 & 56 & 14 & & \\
\hline
\end{tabular}

Table III. Univariate and multivariate analysis of factors influencing the overall survival rate of colorectal cancer patients.

\begin{tabular}{|c|c|c|c|c|c|c|}
\hline \multirow[b]{2}{*}{ Variable } & \multicolumn{3}{|c|}{ Univariate analysis } & \multicolumn{3}{|c|}{ Multivariate analysis } \\
\hline & HR & $95 \% \mathrm{CI}$ & P-value & HR & $95 \% \mathrm{CI}$ & P-value \\
\hline $\mathrm{N}$-cadherin expression & 3.209 & $1.651-6.238$ & 0.001 & 2.672 & $1.241-5.753$ & 0.012 \\
\hline E-cadherin expression & 2.229 & $1.180-4.214$ & 0.014 & 2.135 & $1.085-4.203$ & 0.028 \\
\hline Age & 1.226 & $0.718-2.094$ & 0.456 & 1.286 & $0.734-2.253$ & 0.378 \\
\hline Gender & 0.964 & $0.572-1.626$ & 0.892 & 0.795 & $0.454-1.392$ & 0.422 \\
\hline Tumor location & 0.936 & $0.558-1.571$ & 0.803 & 1.047 & $0.598-1.833$ & 0.871 \\
\hline Tumor differentiation & 1.806 & $1.025-3.182$ & 0.041 & 0.881 & $0.463-1.679$ & 0.701 \\
\hline Tumor size & 0.698 & $0.408-1.193$ & 0.189 & 0.451 & $0.246-0.826$ & 0.100 \\
\hline Tumor invasion & 1.806 & $1.049-3.109$ & 0.033 & 1.321 & $0.723-2.417$ & 0.366 \\
\hline Lymph node metastasis & 1.806 & $1.060-3.076$ & 0.030 & 1.362 & $0.763-2.431$ & 0.296 \\
\hline Distant metastasis & 2.092 & $1.232-3.552$ & 0.006 & 1.923 & $1.081-3.421$ & 0.026 \\
\hline
\end{tabular}

CI, confidence interval; HR, hazard ratio.

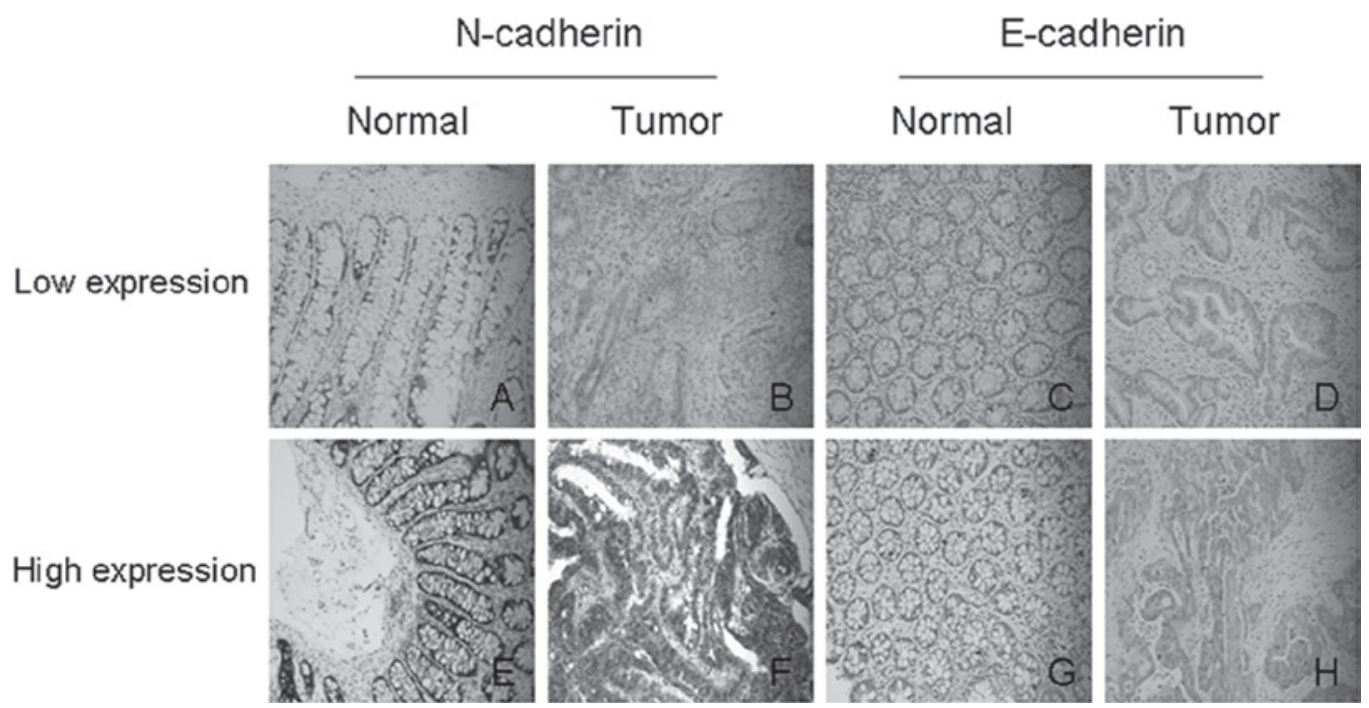

Figure 2. Representative images of immunohistochemical staining. (A) Low expression of N-cadherin in normal tissues. (B) Low expression of N-cadherin in CRC tissues. (C) Low expression of E-cadherin in normal tissues. (D) Low expression of E-cadherin in CRC tissues. (E) High expression of N-cadherin in normal tissues. (F) High expression of N-cadherin in CRC tissues. (G) High expression of E-cadherin in normal tissues. (H) High expression of E-cadherin in CRC tissues (original magnification, $\mathrm{x} 200$ ).

Therefore, the HT-29 cell line was selected for the subsequent assays. Following transfection, the proliferation of the HT-29 cell line was assessed using the MTT method and the results demonstrated that the proliferation of cells in the $\mathrm{N}$-cadherin-silenced group was inhibited compared with the control group transfected with a mock small interfering RNA
(Fig. 4B). Furthermore, the migration of the HT-29 cell line was monitored by wound healing assay and the results indicated that the migration rate in the $\mathrm{N}$-cadherin-silenced group was significantly lower than that in the control-transfected group (Fig. 4C). Of note, following silencing of N-cadherin in the HT-29 cell line, the expression of E-cadherin was 
A

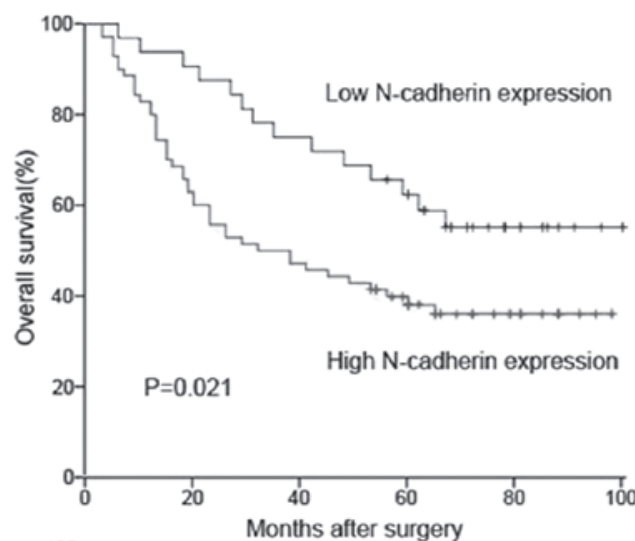

C

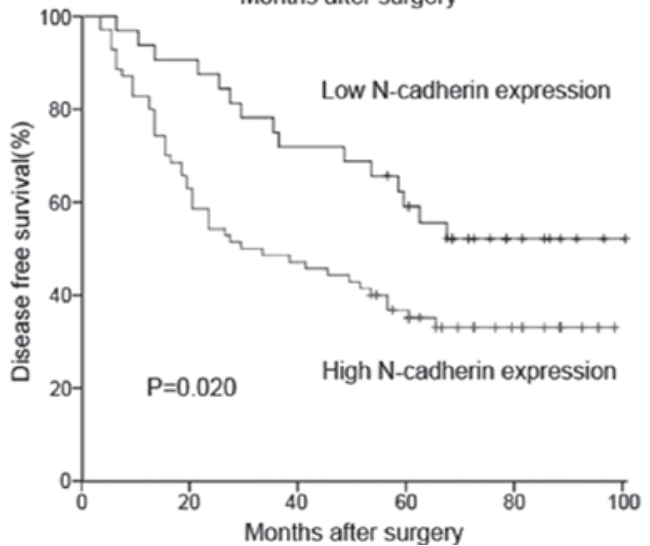

B

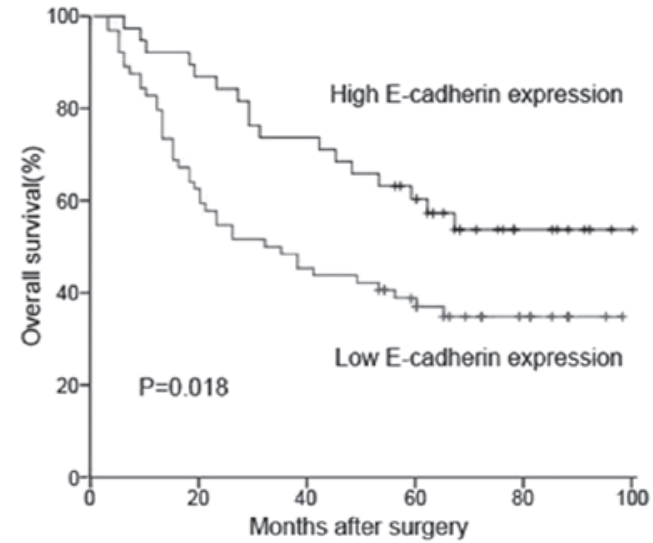

D

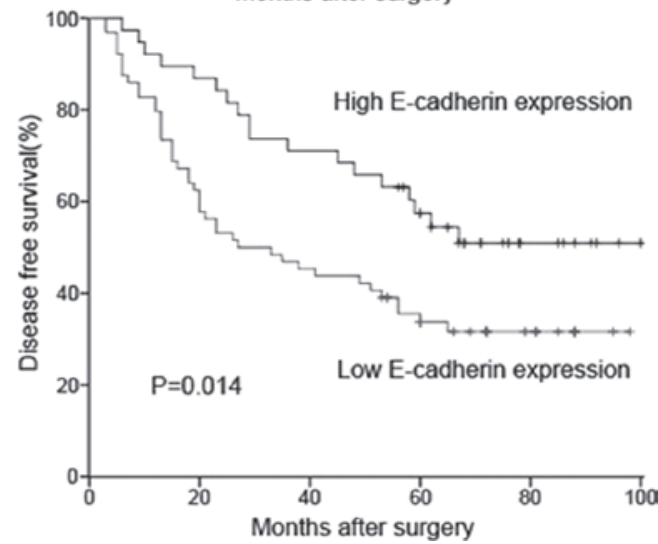

Figure 3. Overall survival (OS) and disease-free survival (DFS) curves of CRC patients according to protein staining. (A) OS curves of CRC patients based on N-cadherin expression. (B) DFS curves of CRC patients based on N-cadherin expression. (C) OS curves of CRC patients based on E-cadherin expression. (D) DFS curves of CRC patients based on E-cadherin expression. The CRC patients with high N-cadherin expression have a significantly lower OS and DFS rate than that with low $\mathrm{N}$-cadherin expression, while the CRC patients with high E-cadherin expression have the opposite result.

A

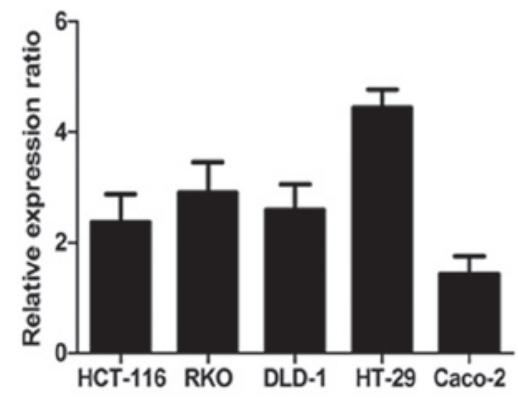

C

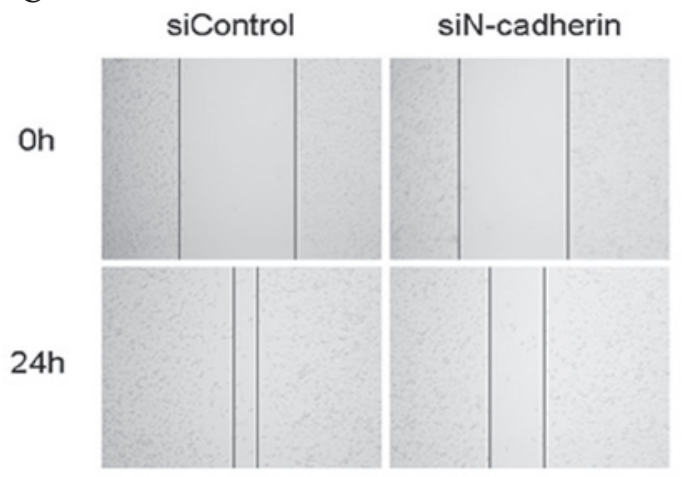

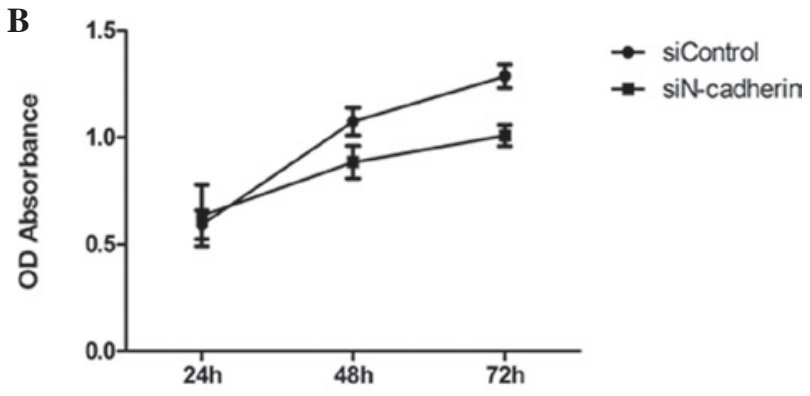

D

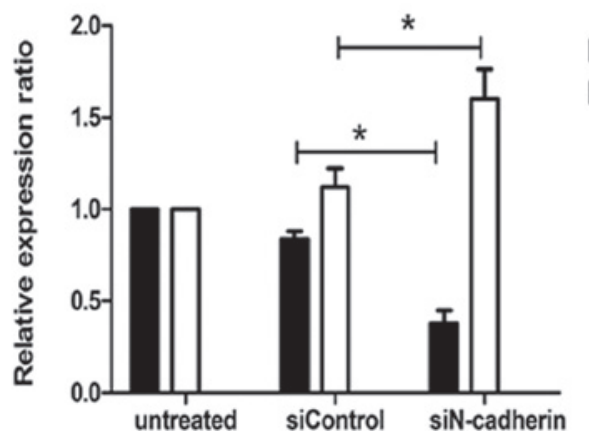

$\mathrm{N}$-cadherin

E-cadherin

Figure 4. Silencing of N-cadherin inhibits proliferation and migration of the CRC cell line HT-29 via up-regulation of E-cadherin. (A) N-cadherin was widely expressed in CRC cell lines, with the highest expression in HT-29 cells. (B) MTT assays showed that the proliferation of HT-29 cells was impaired by silencing of N-cadherin. (C) A wound healing assay showed that the migration ability of HT-29 cells was suppressed following N-cadherin silencing. (D) The mRNA expression of E-cadherin was up-regulated following $\mathrm{N}$-cadherin silencing $\left(\mathrm{N}\right.$-cadherin, ${ }^{*} \mathrm{P}=0.0008 ; \mathrm{E}$-cadherin, ${ }^{*} \mathrm{P}=0.0119$ compared with siControl). siControl, transfected with mock small interfering RNA; siN-cadherin, transfected with N-cadherin small interfering RNA; OD, optical density. 
upregulated (Fig. 4D). This interesting observation confirmed the results of the immunohistochemical assays and suggested that $\mathrm{N}$-cadherin may promote the initiation and development of CRC partly by inducing the EMT.

\section{Discussion}

The development of CRC is a multistep process involving mutational inactivation of tumor-suppressor genes and activation of oncogene pathways (19). A comprehensive understanding of CRC on a molecular level is of great benefit for early detection, targeted therapy and prognosis. Emerging studies have suggested the EMT as an important molecular mechanism underlying the initiation and progression of CRC (20). During the progression of the EMT, cell-cell junctions are lost and epithelial markers such as E-cadherin are replaced by $\mathrm{N}$-cadherin and vimentin, which are commonly defined as mesenchymal markers (21). Despite the increasing number of studies on the tumorigenic roles of mesenchymal markers, knowledge on their roles in CRC remains far from sufficient. Toiyama et al (22) analyzed the vimentin expression in surgical tissue specimens and found that high expression of vimentin was significantly associated with poor disease-free survival of CRC patients. However, with regard to N-cadherin, only a few studies are available on its clinical significance and less is known about its specific role in CRC $(12,13)$.

The present study showed that the expression of $\mathrm{N}$-cadherin was significantly higher in CRC tissues than that in normal tissues. Further experiments demonstrated that $\mathrm{N}$-cadherin expression was associated with tumor differentiation, tumor size as well as tumor, nodes and metastasis stage, suggesting a potential role of $\mathrm{N}$-cadherin in CRC progression. It was also found that OS and DFS were better in patients with low $\mathrm{N}$-cadherin expression than those in patients with high $\mathrm{N}$-cadherin expression. Through multivariate analysis, it was found that high $\mathrm{N}$-cadherin expression was an independent prognostic factor for CRC patients. These results are consistent with those of previous studies on other tumor types, reporting that high expression of $\mathrm{N}$-cadherin was able to predict poor prognosis in bladder cancer (12) and gallbladder cancer (13). Furthermore, the present study also investigated the expression of E-cadherin in CRC tissues and explored the possible association between E-cadherin and $\mathrm{N}$-cadherin. Correlation analysis suggested that $\mathrm{N}$-cadherin expression was negatively associated with E-cadherin expression, implying a possible role of N-cadherin in inducing the EMT of CRC.

To investigate the specific biological role of $\mathrm{N}$-cadherin in CRC cells, RNA interference was employed to downregulate $\mathrm{N}$-cadherin expression in the HT-29 cell line. Of note, following $\mathrm{N}$-cadherin silencing, the proliferative and migratory ability of HT-29 cells was impaired, indicating that $\mathrm{N}$-cadherin may induce metastatic potential in CRC cells. Moreover, E-cadherin expression was up-regulated following $\mathrm{N}$-cadherin silencing, which confirmed the results of the immunohistochemical analysis. Loss of E-cadherin is the best-characterized marker of the EMT, and therefore, the results indicated that $\mathrm{N}$-cadherin may promote $\mathrm{CRC}$ progression partly by inducing EMT. This deduction is also in accordance with a study by Zhang et al (17), which reported that $\mathrm{N}$-cadherin accelerated the proliferation and invasion of erlotinib-resistant lung cancer cell lines by inducing the EMT. Another supporting study also demonstrated that $\mathrm{N}$-cadherin overexpression promotes growth, invasion and metastasis in castration-resistant prostate cancer via EMT (23). In spite of the increasing number of studies on the biological roles of $\mathrm{N}$-cadherin in cancer cells, the present study was the first, to the best of our knowledge, to demonstrate that $\mathrm{N}$-cadherin promotes growth and migration of CRC cells.

Since N-cadherin is regarded as a versatile oncoprotein due to its stimulation of proliferation, invasion, metastasis and angiogenesis (24), pre-clinical tests using $\mathrm{N}$-cadherin interference in animal models have been performed on several tumor types. Su et al (25) found that interference with N-cadherin expression by a monoclonal antibody can effectively prolong survival in a spontaneous highly metastatic pancreatic cancer model. ADH-1, a novel pentapeptide antagonizing $\mathrm{N}$-cadherin, has been proved to markedly augment the anti-tumor effects of chemotherapy in animal models of melanoma (26). Of note, although ADH-1 has been recently evaluated in phase I clinical trials on patients with advanced tumors and has achieved satisfactory responses $(27,28)$, it was also reported to promote tumor growth by activating AKT signaling (29). Therefore, further studies based on cellular and animal models for investigating the complex interaction between tumor cells and targeted treatment for $\mathrm{N}$-cadherin are required.

In summary, the present study demonstrated that $\mathrm{N}$-cadherin expression was upregulated in CRC tissues and negatively correlated with E-cadherin expression. Furthermore, high expression of $\mathrm{N}$-cadherin may be an independent prognostic factor for CRC patients. Moreover, in vitro assays showed that $\mathrm{N}$-cadherin may promote proliferation and migration of CRC cells, partly by inducing the EMT. In conclusion, these findings suggested that $\mathrm{N}$-cadherin is an effective potential target for the treatment of CRC. However, further cellular assays and animal models studies are required to explore the specific role of $\mathrm{N}$-cadherin in CRC initiation and development.

\section{Acknowledgements}

This study was supported by the funding of Science and Technology Commission of Shanghai Municipality (no. 124119a720). The authors would like to thank Professor Yu-Ping Gao (Department of Pathology, Renji Hospital, School of Medicine, Shanghai Jiao Tong University) for her technical assistance in the present study.

\section{References}

1. Jemal A, Bray F, Center MM, Ferlay J, Ward E and Forman D: Global cancer statistics. CA Cancer J Clin 61: 69-90, 2011.

2. Siegel R, Ma J, Zou Z and Jemal A: Cancer statistics, 2014. CA Cancer J Clin 64: 9-29, 2014.

3. Van Cutsem E and Oliveira J; ESMO Guidelines Working Group: Advanced colorectal cancer: ESMO clinical recommendations for diagnosis, treatment and follow-up. Ann Oncol 20 (Suppl 4): 61-63, 2009.

4. Jemal A, Siegel R, Ward E, Hao Y, Xu J and Thun MJ: Cancer statistics, 2009. CA Cancer J Clin 59: 225-249, 2009.

5. Hanahan D and Weinberg RA: Hallmarks of cancer: the next generation. Cell 144: 646-674, 2011.

6. Wheelock MJ, Shintani Y, Maeda M, Fukumoto Y and Johnson KR: Cadherin switching. J Cell Sci 121 (Pt 6): 727-735, 2008. 
7. Le Bras GF, Taubenslag KJ and Andl CD: The regulation of cell-cell adhesion during epithelial-mesenchymal transition, motility and tumor progression. Cell Adh Migr 6: 365-373, 2012.

8. He X, Chen Z, Jia M and Zhao X: Downregulated E-cadherin expression indicates worse prognosis in asian patients with colorectal cancer: Evidence from meta-analysis. PLoS One 8: e70858, 2013.

9. Lee SH, Kim H, Hwang JH, et al: CD24 and S100A4 expression in resectable pancreatic cancers with earlier disease recurrence and poor survival. Pancreas 43: 380-388, 2014.

10. Huang Z, Zhang N, Zha L, et al: Aberrant expression of the autocrine motility factor receptor correlates with poor prognosis and promotes metastasis in gastric carcinoma. Asian Pac J Cancer Prev 15: 989-997, 2014.

11. Fan M, Liu Y, Xia F, et al: Increased expression of EphA2 and E-N cadherin switch in primary hepatocellular carcinoma. Tumori 99: 689-996, 2013.

12. Lascombe I, Clairotte A, Fauconnet S, Bernardini S, Wallerand H, Kantelip B and Bittard $\mathrm{H}$ : N-cadherin as a novel prognostic marker of progression in superficial urothelial tumors. Clin Cancer Res 12:2780-2787, 2006

13. Yi S, Yang ZL, Miao X, et al: N-cadherin and P-cadherin are biomarkers for invasion, metastasis and poor prognosis of gallbladder carcinomas. Pathol Res Pract 210: 363-368, 2014

14. Yamauchi M, Yoshino I, Yamaguchi R, et al: N-cadherin expression is a potential survival mechanism of gefitinib-resistant lung cancer cells. Am J Cancer Res 1: 823-833, 2011.

15. Gao P, Xing AY, Zhou GY, et al: The molecular mechanism of microRNA-145 to suppress invasion-metastasis cascade in gastric cancer. Oncogene 32: 491-501, 2013.

16. Hulit J, Suyama K, Chung S, et al: N-cadherin signaling potentiates mammary tumor metastasis via enhanced extracellular signal-regulated kinase activation. Cancer Res 67: 3106-3116, 2007.

17. Zhang X, Liu G, Kang Y, Dong Z, Qian Q and Ma X: N-cadherin expression is associated with acquisition of EMT phenotype and with enhanced invasion in erlotinib-resistant lung cancer cell lines. PLoS One 8: e57692, 2013.

18. Liang CC, Park AY and Guan JL: In vitro scratch assay: a convenient and inexpensive method for analysis of cell migration in vitro. Nat Protoc 2: 329-333, 2007
19. Markowitz SD and Bertagnolli MM: Molecular origins of cancer: Molecular basis of colorectal cancer. N Engl J Med 361: 2449-2460, 2009.

20. Findlay VJ, Wang C, Watson DK and Camp ER: Epithelial-to-mesenchymal transition and the cancer stem cell phenotype: insights from cancer biology with therapeutic implications for colorectal cancer. Cancer Gene Ther 21: 181-187, 2014.

21. Micalizzi DS, Farabaugh SM and Ford HL: Epithelial-mesenchymal transition in cancer: parallels between normal development and tumor progression. J Mammary Gland Biol Neoplasia 15: 117-134, 2010.

22. Toiyama Y, Yasuda H, Saigusa S, Tanaka K, Inoue Y, Goel A and Kusunoki M: Increased expression of Slug and Vimentin as novel predictive biomarkers for lymph node metastasis and poor prognosis in colorectal cancer. Carcinogenesis 34: 2548-2557, 2013.

23. Tanaka H, Kono E, Tran CP, et al: Monoclonal antibody targeting of $\mathrm{N}$-cadherin inhibits prostate cancer growth, metastasis and castration resistance. Nat Med 16: 1414-1420, 2010.

24. Mariotti A, Perotti A, Sessa C and Rüegg C: N-cadherin as a therapeutic target in cancer. Expert Opin Investig Drugs 16: 451-465, 2007.

25. Su Y, Li J, Witkiewicz AK, Brennan D, Neill T, Talarico J and Radice GL: N-cadherin haploinsufficiency increases survival in a mouse model of pancreatic cancer. Oncogene 31: 4484-4489, 2012.

26. Augustine CK, Yoshimoto Y, Gupta M, et al: Targeting $\mathrm{N}$-cadherin enhances antitumor activity of cytotoxic therapies in melanoma treatment. Cancer Res 68: 3777-3784, 2008.

27. Perotti A, Sessa C, Mancuso A, et al: Clinical and pharmacological phase I evaluation of Exherin ${ }^{\mathrm{TM}}(\mathrm{ADH}-1)$, a selective anti-N-cadherin peptide in patients with $\mathrm{N}$-cadherin-expressing solid tumours. Ann Oncol 20: 741-745, 2009.

28. Yarom N, Stewart D, Malik R, Wells J, Avruch L and Jonker DJ: Phase I clinical trial of Exherin (ADH-1) in patients with advanced solid tumors. Curr Clin Pharmacol 8: 81-88, 2013.

29. Turley RS, Tokuhisa Y, Toshimitsu H, et al: Targeting N-cadherin increases vascular permeability and differentially activates AKT in melanoma. Ann Surg 261, 368-377, 2015. 